\title{
Incidence of infections associated with oral glucocorticoid dose in people diagnosed with polymyalgia rheumatica or giant cell arteritis: a cohort study in England
}

\author{
Jianhua Wu PhD, Adam Keeley BSc, Christian Mallen PhD, Ann W. Morgan PhD, Mar Pujades-Rodriguez PhD MSc
}

Cite as: CMAJ 2019 June 24;191:E680-8. doi: 10.1503/cmaj.190178

\begin{abstract}
BACKGROUND: Most patients with polymyalgia rheumatica or giant cell arteritis are treated with glucocorticoid therapy in primary care. We estimated dose-response risks of infection for this population in England.
\end{abstract}

METHODS: We conducted a retrospective record-linkage study involving a cohort of people with polymyalgia rheumatica or giant cell arteritis registered in family practices across England (1998-2017). Estimates of first occurring infection per level of timevariant current and cumulative dose were obtained using Kaplan-Meier methods and multilevel proportionalhazards Cox models.

RESULTS: Of 39938 patients attending 389 family practices, 22234 (55.7\%) had at least 1 infection over a median follow-up period of 4.8 years, with $5937(26.7 \%)$ requiring hospital admission and 1616 (7.3\%) dying within 7 days of diagnosis. Cumulative risks of all-cause infection were $18.3 \%$ (95\% confidence interval [CI] $17.9 \%-18.7 \%)$ at 1 year, $54.7 \%(95 \%$ $\mathrm{Cl} 54.1 \%-55.2 \%)$ at 5 years and $76.9 \%$ (95\% $\mathrm{Cl} 76.2 \%-77.5 \%)$ at 10 years. Lower respiratory tract infections, conjunctivitis and herpes zoster were the most commonly diagnosed infections. The increases in adjusted hazard ratios (HRs) for all-cause infection per $5 \mathrm{mg}$ prednisolone-equivalent daily dose increase and per $1000 \mathrm{mg}$ cumulative dose increase in the last year from the patient's end date of follow-up were $1.13(95 \% \mathrm{Cl} 1.12-$ $1.14)$ and $1.50(95 \% \mathrm{Cl} 1.49-1.52)$, respectively. Adjusted HRs associated with periods of current glucocorticoid versus no glucocorticoid use ranged from 1.48 (95\% Cl 1.39-1.57) for fungal to $1.70(95 \% \mathrm{Cl} 1.60-1.80)$ for bacterial infection. Stepwise dose-related associations were found for bacterial, viral, parasitic and fungal infections, irrespective of patient age, duration of underlying chronic disease and baseline vaccination status.

INTERPRETATION: We quantified the excess risk of all-cause, bacterial, viral, parasitic and fungal infection conferred by oral glucocorticoids in people with polymyalgia rheumatica or giant cell arteritis and found strong dose responses for all types, even at daily doses of less than $5 \mathrm{mg}$ prednisolone.

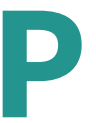

olymyalgia rheumatica and giant cell arteritis are chronic inflammatory diseases requiring immunosuppressive therapy with glucocorticoids to induce remission and treat subsequent episodic flares. Most patients with polymyalgia rheumatica or giant cell arteritis are treated with medium-to-high doses of prednisolone (up to $15 \mathrm{mg}$ for polymyalgia rheumatica and $60 \mathrm{mg}$ for giant cell arteritis) for 6 months to several years. ${ }^{1,2}$ Glucocorticoids are effective in reducing symptoms and inflammation associated with both diseases, but their immunosuppressive effects ${ }^{3-5}$ are known to increase the risk of infection.
Dose-response estimates of absolute and relative risks of infections in people diagnosed with polymyalgia rheumatica or giant cell arteritis are lacking, despite these conditions being the leading indications for long-term glucocorticoid prescription in primary care. ${ }^{1,2}$ It is uncertain whether the magnitude of dose-response risks varies for different types of infections. ${ }^{6}$

Previous studies have provided evidence of the increased risk of serious and opportunistic infection in patients with autoimmune diseases treated with high doses of glucocorticoids. ${ }^{7-9}$ Most were observational studies that examined risks of specific infections, such as septicemia and lower respiratory 
tract infections, and most were conducted among patients with rheumatoid arthritis, aged 65 years and older, and examined risks of severe infections requiring hospital admission or accident and emergency services. ${ }^{7,8,10-12}$ Findings from a recent cohort study suggest that the risk of infection in patients treated with oral glucocorticoids may vary for different types of autoimmune diseases. ${ }^{13}$

For patients with polymyalgia rheumatica or giant cell arteritis, for whom current therapeutic options are limited, adequate characterization of glucocorticoid dose-response relations is required to conduct benefit-harm assessments and cost-effectiveness studies. These results will help guide the introduction of newly licensed glucocorticoid-sparing drugs, such as tocilizumab, into clinical practice.

We aimed to assess the risk of all-cause, bacterial, viral, parasitic and fungal infections associated with current daily and cumulative dose of oral glucocorticoids in a large cohort of people diagnosed with polymyalgia rheumatica or giant cell arteritis in England.

\section{Methods}

\section{Study design and follow-up}

This was a retrospective cohort study conducted among adults attending Clinical Practice Research Datalink (CPRD) family practices in England that consented to data linkage to hospital databases. All patients continuously registered in their family practice for 1 year or longer between Jan. 1, 1997, and Mar. 15, 2017, aged 18 years and older, and with a diagnosis of polymyalgia rheumatica or giant cell arteritis were eligible for inclusion (Supplemental Figure 1, Appendix 1, available at www.cmaj.ca/lookup/suppl/doi:10.1503/cmaj.190178/-/ DC1). Patients with prevalent and incident polymyalgia rheumatica or giant cell arteritis were included. For each patient, follow-up started on the earliest date on which all the eligibility criteria were met and ended on the earliest of the following dates: first occurrence of the outcome analyzed, death, date leaving the practice or last date of data collection in the practice.

\section{Data sources}

We analyzed 3 linked data sources (Appendix 2, available at www.cmaj.ca/lookup/suppl/doi:10.1503/cmaj.190178/-/DC1). Primary health care records from CPRD contained demographic and health behaviour data, diagnosed diseases (e.g., infections), prescribed medication, and results of laboratory and clinical examinations. ${ }^{14}$ Patients are broadly representative of the population in England in terms of age, sex and ethnicity. ${ }^{14,15}$ Validation studies have provided evidence of the accuracy of diagnostic and prescribing information recorded. ${ }^{14}$ Hospital records from the Hospital Episode Statistics contained diagnoses recorded during hospital admissions for patients receiving care funded by the National Health Service in England. ${ }^{16}$ We used data from the mortality registry to identify dates and causes of death. Patients with polymyalgia rheumatica or giant cell arteritis were identified with codes previously used and validated (Read Version 2: G755100, G755000, G755.00, Nyu4100, G755z00, G755200, N20..00, N20..11, N200.00; International Classification of Diseases, 10th Revision: M31.5, M31.6, M35.3). ${ }^{17,18}$ The positive predictive value for giant cell arteritis in CPRD is $91 \% .^{18}$

\section{Oral glucocorticoid exposure}

For each prescription of oral glucocorticoids, the daily dose was derived from recorded information on the product strength (e.g., $1 \mathrm{mg}$ ), directions given (e.g., 1 tablet a day) and quantity prescribed (e.g., 28 tablets). We derived the duration of each prescription by dividing the daily dose by the prescribed total dose. The daily dose was converted into milligrams of prednisolone-equivalent dose (Supplemental Table 1, Appendix 3, available at www.cmaj.ca/lookup/suppl/ doi:10.1503/cmaj.190178/-/DC1) to account for variation in the relative antiinflammatory effects of different types of glucocorticoids.

We predefined several time-variant glucocorticoid exposure variables (Supplemental Figure 2, Appendix 1): 1) binary variable for current use (e.g., whether the patient received glucocorticoids at a given time point or not); 2) current daily dose per $5 \mathrm{mg} / \mathrm{d}$ (zero when medication was not prescribed), considered as continuous and categorical (nonuse, $>$ 0.0-4.9 mg, 5.0-14.9 mg, 15.0-24.9 mg, $\geq 25.0 \mathrm{mg} / \mathrm{d}$ ) variables; 3 ) cumulative dose since 1 year before follow-up start per $1000 \mathrm{mg}$, calculated by summing the total dose prescribed up to that point and dividing it by 1000 (this was also considered as continuous and categorical [nonuse, > 0-959 mg, 960$3054 \mathrm{mg}, 3055-7299 \mathrm{mg}, \geq 7300 \mathrm{mg}$, cutoffs as defined by Movahedi and colleagues ${ }^{19}$ ] variables); and 4) cumulative dose 1 year before the end of follow-up, calculated by summing the total dose from 1 year before the end of follow-up or the whole study period if the duration of follow-up was less than 1 year. To create these variables, we determined the start and end of each medication exposure period and split patient follow-up on the dates on which the dose changed (Appendix 2).

\section{Study outcomes}

The primary outcome was the first occurrence of all-cause infection (Supplemental Figure 3, Appendix 1; Supplemental Table 2, Appendix 3). Secondary outcomes were first occurrence of cause-specific types of infection: bacterial, viral, parasitic and fungal. To capture incident cases of infection, we did not consider episodes from patients with the same type of infection code recorded within 14 days (or 1 year for tuberculosis and hepatitis) before the start of follow-up.

\section{Covariates}

The covariates considered were as follows: baseline age; sex; smoking status; ethnicity; body mass index; socioeconomic status (index of multiple deprivation, ${ }^{20}$ an area-based indicator based on the patient's residence); underlying disease (polymyalgia rheumatica, giant cell arteritis and both); comorbidities 
(cardiovascular disease, diabetes, cancer, asthma, chronic obstructive pulmonary disease, renal disease and HIV); vaccination status (within last year for influenza; at any time before start of follow-up for varicella zoster and pneumococcus); the number of hospital admissions, prescribed non-oral glucocorticoids (inhaled, nasal, parenteral, topical and rectal), $\mathrm{H}_{2}$ antagonists and proton pump inhibitors in the last year; and time-variant prescribed disease-modifying antirheumatic drugs and nonsteroidal antiinflammatory drugs (NSAIDs) during follow-up (Appendix 2).

\section{Statistical analysis}

We report standard descriptive statistics for baseline characteristics. Cumulative probabilities of all-cause and typespecific infection were assessed using Kaplan-Meier methods. We estimated incident rates with $95 \%$ confidence intervals (Cls) by dividing the number of infections by the total number of person-years of follow-up.

We assessed dose-response associations using Cox proportional hazards models, including the practice identifier as a random intercept to account for clustering effect. We estimated adjusted hazard ratios (HRs) with $95 \% \mathrm{Cls}$ for each type of infection regressed on each of the time-variant exposures. Every model was adjusted for the covariates listed and using time-variant prescribed disease-modifying antirheumatic drugs and NSAIDs during follow-up. We assessed the proportional hazards assumption using Schoenfeld residuals tests. Missing daily glucocorticoid dose and baseline covariates were replaced through generation of 25 data sets using multiple imputation with chained equations (Appendix 2). All models were conducted for each imputed data set. We generated pooled estimates and accompanying $95 \% \mathrm{Cls}$ using Rubin's rules.

In secondary analyses, we obtained estimates by sex, duration since diagnosis of polymyalgia rheumatica or giant cell arteritis, age group, baseline vaccination status and use of non-oral glucocorticoids in the last year.

Data management and analyses were performed in Stata version 15 and $\mathrm{R}$ version 3.3.1 (http://cran.r-project.org).

\section{Ethics approval}

Approval for the study was obtained from the Independent Scientific Advisory Committee for Medicines and Healthcare products Regulatory Agency database research.

\section{Results}

A total of 39938 patients attending 389 family practices were included, 32735 (82.0\%) with polymyalgia rheumatica, 4505 $(11.3 \%)$ with giant cell arteritis and 2698 (6.8\%) with both (Table 1). The median time since diagnosis of polymyalgia rheumatica or giant cell arteritis was 4.9 (interquartile range [IQR] 3.0-7.0) years. Of the patients, 12346 (30.9\%) were men, and the median time at risk was 2.4 (IQR 0.9-5.0) years. At the start of follow-up, the mean patient age was 73.0 (standard deviation 10.7) years, 16966 (42.5\%) were nonsmokers, 19515 (48.9\%) were vaccinated against pneumococcus, 25640 (64.2\%) were vaccinated against influenza, and $7676(19.2 \%)$ received inhaled or nasal glucocorticoids (Supplemental Tables 3 and 4, Appendix 3). At baseline, the most common diagnosed comorbidities were cardiovascular disease (32.7\%), asthma (15.9\%) and diabetes (12.6\%).

The median glucocorticoid cumulative dose received during follow-up was 3451.5 (IQR 1606.5-6465.0) $\mathrm{mg}$ and in the last year of follow-up was 1598.4 (IQR 780.0-2552.7) mg. During follow-up, 3809 (9.5\%) patients received a disease-modifying antirheumatic drug.

\section{Incidence, type and severity of infection}

A total of 22234 (55.7\%) people had an infection during 138412 person-years of follow-up; the median duration of follow-up per patient was 4.8 (IQR 2.2-8.5) years. The incidence of all-cause infection was 160.7 (95\% Cl 159.3-162.2) per 1000 person-years (Supplemental Table 5, Appendix 3). Types of infection most frequently diagnosed were lower respiratory tract infections (6064 [27.3\%]), conjunctivitis (1905 [8.6\%]) and herpes zoster (1642 [7.4\%]) (Supplemental Table 6, Appendix 3). Of the 22234 patients who had an infection, $5937(26.7 \%)$ were admitted to hospital on the date of or within 7 days after infection diagnosis, 1930 (8.7\%) died within 30 days of diagnosis and 1616 (7.3\%) died within 7 days of diagnosis. The most common causes of death from infection were pneumonia (1015 [52.6\%]), urinary tract infection (58 [3.0\%]) and peritonitis (43 [2.2\%]).

The cumulative probabilities of all types of infection increased with higher daily dose (Table 2). At 1 year, probabilities of all-cause infection increased from $12.9 \%(95 \% \mathrm{Cl}$ $12.4 \%-13.4 \%)$ for periods of nonuse, through $18.0 \%(95 \% \mathrm{Cl}$ $17.1 \%-18.8 \%)$ for a dose between greater than 0.0 and $4.9 \mathrm{mg} / \mathrm{d}$, to $35.8 \%(95 \% \mathrm{Cl} 32.9 \%-38.6 \%)$ for $25.0 \mathrm{mg} / \mathrm{d}$ or greater.

\section{Dose-response associations}

Compared with periods of nonuse of oral glucocorticoids, adjusted HRs of all types of infection were higher in periods of current use (adjusted HR 1.49, 95\% Cl 1.44-1.53 for allcause infection; Table 3$)$. The highest estimates were seen for bacterial infections (adjusted HR 1.70, 95\% Cl 1.60-1.80) and the lowest for fungal infections (adjusted HR $1.48,95 \% \mathrm{Cl}$ 1.39-1.57). Compared with nonuse of oral glucocorticoids, the increase in adjusted HR per $5 \mathrm{mg}$ daily dose was 1.13 $(95 \% \mathrm{Cl} 1.12-1.14)$ for all-cause infection, with adjusted HRs increasing from $1.39(95 \% \mathrm{Cl} 1.34-1.45)$ for a dose between greater than 0.0 and $4.9 \mathrm{mg} / \mathrm{d}$ to $2.30(95 \% \mathrm{Cl} 2.13-2.49)$ for $25 \mathrm{mg} / \mathrm{d}$ or greater. The increase in adjusted HR per $1000 \mathrm{mg}$ cumulative dose during the last year was $1.50(95 \% \mathrm{Cl}$ 1.49-1.52).

Secondary analyses showed similar dose-response estimates regardless of sex and time since diagnosis of the underlying disease (Table 4; Supplemental Tables 7 and 8, Appendix 3; Appendix 4, available at www.cmaj.ca/lookup/suppl/ doi:10.1503/cmaj.190178/-/DC1). Higher cumulative probabilities 
of all-cause and bacterial infection were found in older patients (Supplemental Table 9, Appendix 3). Patients who were vaccinated had higher risks of all types of infections than those not vaccinated. Patients prescribed non-oral glucocorticoids had higher risks of all types of infections than those not treated with non-oral glucocorticoids (Supplemental Tables 10-12, Appendix 3).

\section{Interpretation}

In this population-based study including 39938 patients diagnosed with polymyalgia rheumatica or giant cell arteritis, we found high absolute risks of all types of infection and a marked stepwise increase in risk with higher oral glucocorticoid doses. In periods with prescribed medication, patients'

Table 1: Baseline characteristics of adult patients attending 389 family practices in England, by disease type

No. $(\%)$ of patients or mean $\pm S D^{*}$

\begin{tabular}{|c|c|c|c|c|}
\hline Characteristic & $\begin{array}{c}\text { Total } \\
n=39938\end{array}$ & $\begin{array}{l}\text { GCA only } \\
n=4505\end{array}$ & $\begin{array}{l}\text { PMR only } \\
n=32735\end{array}$ & $\begin{array}{c}\text { PMR and GCA } \\
n=2698\end{array}$ \\
\hline \multicolumn{5}{|l|}{ Sociodemographic information } \\
\hline Sex, male & $12346(30.9)$ & $1391(30.9)$ & $10373(31.7)$ & $582(21.6)$ \\
\hline Age, yr & $73.0 \pm 10.7$ & $71.1 \pm 12.6$ & $73.2 \pm 10.5$ & $73.9 \pm 8.7$ \\
\hline \multicolumn{5}{|l|}{ Ethnicity† } \\
\hline White & $35692(89.4)$ & $4001(88.8)$ & 29205 (89.2) & $2486(92.1)$ \\
\hline Other & $959(2.4)$ & $220(4.9)$ & $671(2.0)$ & $68(2.5)$ \\
\hline \multicolumn{5}{|l|}{ Index of Multiple Deprivation (fifths) } \\
\hline First (least deprived) & $7583(19.0)$ & $709(15.7)$ & $6358(19.4)$ & $516(19.1)$ \\
\hline Fifth (most deprived) & $5311(13.3)$ & $829(18.4)$ & $4104(12.5)$ & $378(14.0)$ \\
\hline \multicolumn{5}{|l|}{ Biomarkers } \\
\hline Body mass index $\dagger$ & $27.5 \pm 5.6$ & $27.3 \pm 6.0$ & $27.5 \pm 5.5$ & $26.9 \pm 5.7$ \\
\hline C-reactive protein, $\mathrm{mg} / \mathrm{L}$ & $33.1 \pm 43.4$ & $36.7 \pm 57.2$ & $32.2 \pm 40.8$ & $40.0 \pm 53.6$ \\
\hline Erythrocyte sedimentation rate, $\mathrm{mm} / \mathrm{h}$ & $40.4 \pm 29.0$ & $41.4 \pm 32.5$ & $39.9 \pm 28.2$ & $45.2 \pm 32.1$ \\
\hline Total white blood cells, $\times 10^{9} / \mathrm{L}$ & $8.7 \pm 2.7$ & $8.5 \pm 3.0$ & $8.7 \pm 2.7$ & $9.0 \pm 2.9$ \\
\hline Neutrophils, $\times 10^{9} / \mathrm{L}$ & $5.8 \pm 2.4$ & $5.7 \pm 2.7$ & $5.8 \pm 2.3$ & $6.2 \pm 2.6$ \\
\hline Lymphocytes, $x 10^{9} / \mathrm{L}$ & $2.0 \pm 0.9$ & $2.0 \pm 1.0$ & $2.0 \pm 0.9$ & $1.9 \pm 0.9$ \\
\hline Eosinophils, $\times 10^{9} / \mathrm{L}$ & $0.2 \pm 0.2$ & $0.2 \pm 0.2$ & $0.2 \pm 0.2$ & $0.2 \pm 0.2$ \\
\hline Hemoglobin, g/L & $128 \pm 14$ & $129 \pm 15$ & $128 \pm 14$ & $126 \pm 14$ \\
\hline Creatinine, $\mu \mathrm{mol} / \mathrm{L}$ & $88.8 \pm 33.6$ & $92.2 \pm 49.7$ & $88.5 \pm 31.2$ & $87.0 \pm 29.8$ \\
\hline \multicolumn{5}{|l|}{ Smoking status $\dagger$} \\
\hline Nonsmoker & $16966(42.5)$ & $1710(38.0)$ & $14084(43.0)$ & $1172(43.4)$ \\
\hline Former smoker & $8302(20.8)$ & $824(18.3)$ & $6982(21.3)$ & $496(18.4)$ \\
\hline Current smoker & $8197(20.5)$ & $1221(27.1)$ & $6440(19.7)$ & $536(19.9)$ \\
\hline Duration since first recorded diagnosed disease, yr, median (IQR) & $4.9(3.0-7.0)$ & $4.0(2.0-6.0)$ & $4.9(3.0-7.0)$ & $6.1(2.0-9.0)$ \\
\hline No. of hospital admissions in last year & $0.6 \pm 2.6$ & $0.9 \pm 4.0$ & $0.5 \pm 2.4$ & $0.6 \pm 1.3$ \\
\hline \multicolumn{5}{|l|}{ Comorbidities $\ddagger$} \\
\hline Cardiovascular disease & $13044(32.7)$ & $1649(36.6)$ & $10464(32.0)$ & $931(34.5)$ \\
\hline Diabetes & $5020(12.6)$ & $647(14.4)$ & $4055(12.4)$ & $318(11.8)$ \\
\hline Asthma & $6336(15.9)$ & $817(18.1)$ & $5057(15.4)$ & $462(17.1)$ \\
\hline COPD & $2987(7.5)$ & $466(10.3)$ & $2328(7.1)$ & $193(7.2)$ \\
\hline Cancer & $4050(10.1)$ & $466(10.3)$ & $3348(10.2)$ & $236(8.7)$ \\
\hline Chronic renal disease & $1347(3.4)$ & $163(3.6)$ & $1095(3.3)$ & $89(3.3)$ \\
\hline
\end{tabular}

Note: $\mathrm{COPD}=$ chronic obstructive pulmonary disease, $\mathrm{GCA}=$ giant cell arteritis, $\mathrm{IQR}=$ interquartile range, $\mathrm{PMR}=$ polymyalgia rheumatica, $\mathrm{SD}=$ standard deviation .

†Data on ethnicity, body mass index and smoking status were missing for $8.1 \%, 29.8 \%$ and $16.2 \%$ of patients, respectively.

†Two patients had HIV infection at baseline. 
risk was $50 \%$ higher than when it was not prescribed. Increases in risk ranged from $48 \%$ for fungal to $70 \%$ for bacterial infections. For every increase of $5 \mathrm{mg}$ daily dose and of $1000 \mathrm{mg}$ cumulative dose in the last year, the increased risks of all-cause infection were $13 \%$ and $50 \%$, respectively.
When assessing dose response with total cumulative dose, we found evidence of increased risk only for a dose of $7300 \mathrm{mg}$ or greater. This weaker association is expected given that the risk conferred by glucocorticoids would decrease with time since exposure discontinuation. Dose-response estimates

Table 2 (part 1 of 2): Kaplan-Meier estimates of infection per level of time-variant current daily and cumulative oral glucocorticoid prednisolone-equivalent dose

Cumulative probability, $\%(95 \% \mathrm{CI})^{\star}$; infection type

\begin{tabular}{|c|c|c|c|c|c|}
\hline Variable & All-cause & Bacterial & Viral & Parasitic & Fungal \\
\hline Incident infections, no. (\%) & $22234(100)$ & $5234(23.5)$ & $5344(24.0)$ & $1120(5.0)$ & $5416(24.4)$ \\
\hline At 1 year & $18.3(17.9-18.7)$ & $3.2(3.0-3.3)$ & $3.8(3.6-3.9)$ & $0.9(0.8-1.0)$ & $3.9(3.7-4.1)$ \\
\hline \multicolumn{6}{|l|}{ Current daily dose, mg } \\
\hline Nonuse & $12.9(12.4-13.4)$ & $2.1(1.8-2.3)$ & $2.5(2.2-2.7)$ & $0.6(0.5-0.7)$ & $2.4(2.2-2.7)$ \\
\hline$>0.0-4.9$ & $18.0(17.1-18.8)$ & $3.4(3.0-3.8)$ & $3.6(3.2-4.0)$ & $0.8(0.6-1.0)$ & $4.0(3.6-4.5)$ \\
\hline $5.0-14.9$ & $21.1(20.4-21.8)$ & $3.5(3.1-3.8)$ & $4.6(4.2-5.0)$ & $1.1(0.9-1.3)$ & $4.7(4.3-5.1)$ \\
\hline $15.0-24.9$ & $30.4(28.5-32.2)$ & $6.3(5.3-7.4)$ & $7.2(6.1-8.3)$ & $2.0(1.4-2.5)$ & $7.3(6.3-8.4)$ \\
\hline$\geq 25.0$ & $35.8(32.9-38.6)$ & $7.9(6.2-9.7)$ & $8.0(6.2-9.7)$ & $2.1(1.1-3.0)$ & $8.5(6.7-10.3)$ \\
\hline \multicolumn{6}{|c|}{ Cumulative dose in last year, mg } \\
\hline Nonuse & $5.1(4.8-5.3)$ & $0.7(0.6-0.8)$ & $0.8(0.7-0.9)$ & $0.2(0.1-0.2)$ & $0.9(0.7-1.0)$ \\
\hline$>0.0-959.9$ & $39.0(37.4-40.5)$ & $11.4(10.0-12.7)$ & $13.9(12.5-15.3)$ & $3.6(2.8-4.4)$ & $12.9(11.6-14.3)$ \\
\hline $960.0-3054.9$ & $49.2(47.9-50.4)$ & $17.1(15.7-18.5)$ & $18.5(17.1-19.9)$ & $5.6(4.7-6.5)$ & $20.3(18.9-21.7)$ \\
\hline$\geq 3055.0$ & $60.2(57.9-62.4)$ & $23.3(20.2-26.3)$ & $25.8(22.7-28.7)$ & $7.9(5.9-9.9)$ & $24.4(21.3-27.3)$ \\
\hline \multicolumn{6}{|l|}{ Total cumulative dose, mg } \\
\hline Nonuse & $20.6(19.6-21.6)$ & $3.5(3.0-4.0)$ & $4.0(3.5-4.5)$ & $1.0(0.8-1.3)$ & $4.3(3.8-4.9)$ \\
\hline$>0.0-959.9$ & $29.0(27.8-30.3)$ & $5.4(4.7-6.0)$ & $6.9(6.2-7.6)$ & $1.6(1.2-1.9)$ & $6.6(5.9-7.3)$ \\
\hline $960.0-3054.9$ & $21.9(21.2-22.6)$ & $4.0(3.7-4.4)$ & $4.5(4.2-4.9)$ & $1.1(1.0-1.3)$ & $5.2(4.8-5.6)$ \\
\hline $3055.0-7299.9$ & $10.2(9.7-10.7)$ & $1.8(1.5-2.0)$ & $2.1(1.9-2.4)$ & $0.5(0.4-0.6)$ & $1.8(1.6-2.1)$ \\
\hline$\geq 7300.0$ & $7.4(6.0-8.9)$ & $0.6(0.2-1.0)$ & $1.2(0.7-1.8)$ & $0.4(0.1-0.8)$ & $1.4(0.8-2.0)$ \\
\hline At 5 years & $54.7(54.1-55.2)$ & $11.6(11.2-12.0)$ & $13.1(12.7-13.5)$ & $2.8(2.6-3.0)$ & $13.7(13.3-14.1)$ \\
\hline \multicolumn{6}{|l|}{ Current daily dose, mg } \\
\hline Nonuse & $42.1(41.3-42.9)$ & $7.6(7.2-7.9)$ & $8.8(8.4-9.2)$ & $1.9(1.7-2.1)$ & $9.4(8.9-9.8)$ \\
\hline$>0.0-4.9$ & $65.0(63.6-66.4)$ & $17.0(15.8-18.1)$ & $17.7(16.4-18.8)$ & $3.3(2.7-3.8)$ & $18.5(17.3-19.7)$ \\
\hline $5.0-14.9$ & $69.8(68.7-71.0)$ & $17.6(16.6-18.6)$ & $20.3(19.2-21.4)$ & $4.5(4.0-5.1)$ & $20.5(19.4-21.6)$ \\
\hline $15.0-24.9$ & $77.9(75.4-80.1)$ & $23.7(20.8-26.5)$ & $24.6(21.8-27.4)$ & $6.4(4.8-8.0)$ & $27.7(24.6-30.7)$ \\
\hline$\geq 25.0$ & $81.5(78.2-84.3)$ & $28.4(24.0-32.5)$ & $27.5(23.1-31.6)$ & $7.2(4.6-9.7)$ & $26.7(22.6-30.5)$ \\
\hline \multicolumn{6}{|c|}{ Cumulative dose in last year, mg } \\
\hline Nonuse & $32.6(31.8-33.3)$ & $4.9(4.6-5.2)$ & $5.9(5.6-6.2)$ & $1.1(1.0-1.3)$ & $6.3(5.9-6.6)$ \\
\hline$>0.0-959.9$ & $74.9(73.3-76.3)$ & $24.0(22.1-25.8)$ & $26.1(24.2-27.9)$ & $5.4(4.5-6.4)$ & $26.7(24.9-28.5)$ \\
\hline $960.0-3054.9$ & $84.5(83.4-85.4)$ & $33.1(31.2-35.0)$ & $35.0(33.1-36.9)$ & $9.2(7.9-10.4)$ & $36.9(35.0-38.8)$ \\
\hline$\geq 3055.0$ & $92.7(91.0-94.0)$ & $50.3(45.5-54.8)$ & $47.7(43.0-52.1)$ & $13.4(9.9-16.7)$ & $45.2(40.6-49.5)$ \\
\hline \multicolumn{6}{|l|}{ Total cumulative dose, $\mathrm{mg}$} \\
\hline Nonuse & $58.7(57.1-60.2)$ & $12.3(11.2-13.4)$ & $14.3(13.2-15.5)$ & $3.4(2.8-4.0)$ & $15.6(14.3-16.8)$ \\
\hline$>0.0-959.9$ & $62.4(60.8-64.0)$ & $14.2(13.0-15.4)$ & $17.2(15.9-18.5)$ & $3.9(3.2-4.5)$ & $17.3(16.0-18.6)$ \\
\hline $960.0-3054.9$ & $61.7(60.5-62.8)$ & $14.5(13.7-15.4)$ & $16.1(15.1-17.0)$ & $3.6(3.1-4.1)$ & $18.0(17.1-19.0)$ \\
\hline $3055.0-7299.9$ & $55.2(54.1-56.2)$ & $11.9(11.2-12.6)$ & $13.7(13.0-14.4)$ & $2.8(2.4-3.1)$ & $13.8(13.0-14.5)$ \\
\hline$\geq 7300.0$ & $36.1(34.8-37.3)$ & $7.2(6.6-7.8)$ & $7.4(6.8-8.0)$ & $1.4(1.2-1.7)$ & $7.5(6.9-8.1)$ \\
\hline
\end{tabular}


Table 2 (part 2 of 2): Kaplan-Meier estimates of infection per level of time-variant current daily and cumulative oral glucocorticoid prednisolone-equivalent dose

Cumulative probability, $\%(95 \% \mathrm{Cl}){ }^{\star}$; infection type

\begin{tabular}{|c|c|c|c|c|c|}
\hline Variable & All-cause & Bacterial & Viral & Parasitic & Fungal \\
\hline At 10 years & $76.9(76.2-77.5)$ & $21.0(20.4-21.7)$ & $21.2(20.6-21.8)$ & $4.2(4.0-4.5)$ & $21.2(20.6-21.8)$ \\
\hline \multicolumn{6}{|c|}{ Current daily dose, mg } \\
\hline Nonuse & $68.0(67.1-68.9)$ & $15.6(15.0-16.3)$ & $15.9(15.2-16.6)$ & $3.0(2.7-3.3)$ & $16.2(15.6-16.9)$ \\
\hline$>0.0-4.9$ & $84.4(82.9-85.8)$ & $29.2(27.2-31.1)$ & $27.9(26.0-29.8)$ & $5.3(4.4-6.2)$ & $28.6(26.6-30.4)$ \\
\hline $5.0-14.9$ & $88.7(87.6-89.7)$ & $30.6(28.8-32.4)$ & $30.9(29.2-32.6)$ & $6.8(5.8-7.8)$ & $29.4(27.8-31.1)$ \\
\hline $15.0-24.9$ & $92.3(89.9-94.2)$ & $35.9(31.2-40.2)$ & $38.2(33.2-42.8)$ & $9.6(6.7-12.5)$ & $36.1(31.8-40.1)$ \\
\hline$\geq 25.0$ & $93.1(90.1-95.2)$ & $45.5(37.9-52.2)$ & $39.4(32.5-45.5)$ & $11.6(7.0-15.9)$ & $36.9(30.6-42.6)$ \\
\hline \multicolumn{6}{|c|}{ Cumulative dose in last year, $\mathrm{mg}$} \\
\hline Nonuse & $62.5(61.5-63.5)$ & $12.8(12.1-13.4)$ & $13.1(12.5-13.7)$ & $2.3(2.0-2.5)$ & $13.3(12.7-13.9)$ \\
\hline$>0.0-959.9$ & $88.7(87.1-90.1)$ & $33.1(30.4-35.7)$ & $33.1(30.4-35.7)$ & $6.7(5.4-7.9)$ & $32.9(30.4-35.4)$ \\
\hline $960.0-3054.9$ & $93.1(92.0-94.0)$ & $41.8(39.0-44.4)$ & $40.5(37.8-43.1)$ & $10.4(8.6-2.2)$ & $37.8(35.5-40.1)$ \\
\hline$\geq 3055.0$ & $97.2(95.7-98.2)$ & $54.4(48.4-59.7)$ & $50.7(43.9-56.7)$ & $10.7(6.5-14.6)$ & $47.0(40.6-52.7)$ \\
\hline \multicolumn{6}{|c|}{ Total cumulative dose, $\mathrm{mg}$} \\
\hline Nonuse & $79.3(77.5-80.9)$ & $22.3(20.3-24.2)$ & $23.4(21.5-25.2)$ & $5.2(4.2-6.2)$ & $23.2(21.4-25.0)$ \\
\hline$>0.0-959.9$ & $82.2(80.4-83.8)$ & $22.6(20.7-24.5)$ & $25.8(23.8-27.8)$ & $5.8(4.7-6.8)$ & $26.2(24.2-28.1)$ \\
\hline $960.0-3054.9$ & $81.3(80.0-82.5)$ & $24.2(22.7-25.6)$ & $23.7(22.3-25.1)$ & $5.2(4.5-5.9)$ & $26.0(24.6-27.4)$ \\
\hline $3055.0-7299.9$ & $76.0(74.8-77.2)$ & $21.0(19.8-22.1)$ & $21.6(20.4-22.7)$ & $4.0(3.5-4.6)$ & $21.5(20.4-22.7)$ \\
\hline$\geq 7300.0$ & $67.4(65.9-68.8)$ & $17.4(16.3-18.4)$ & $16.1(15.1-17.1)$ & $2.9(2.5-3.3)$ & $14.8(13.8-15.8)$ \\
\hline
\end{tabular}

Note: $\mathrm{Cl}=$ confidence interval.

*Unless stated otherwise.

were similar regardless of sex, disease subtype (i.e., polymyalgia rheumatica, giant cell arteritis or both), and disease duration. However, higher dose-response risks of all infections were observed for patients prescribed than for those not prescribed non-oral glucocorticoids, and higher risks of bacterial infections were found in older patients than in younger patients. Higher dose-response risks were also found for patients vaccinated against pneumococcus, influenza and varicella zoster than for patients not vaccinated. This is consistent with patients with high morbidity burden (e.g., older patients) being more often vaccinated and having high risk for infections for which they are unprotected.

A previous study conducted among 1664 patients with giant cell arteritis reported adjusted rate ratios of lower respiratory tract infections, upper urinary tract infections and sepsis for oral glucocorticoid users, compared with matched controls. ${ }^{9}$ Studies among patients with rheumatoid arthritis have reported increased risk of common serious infections (e.g., bacterial pneumonia and herpes zoster $)^{8,10-12,21}$ but not of nonserious infections. ${ }^{21}$ In contrast, in a large case-control study involving patients with rheumatoid arthritis, the risk of nonserious infections was higher in patients receiving oral glucocorticoids, with adjusted HRs increasing from 1.10 for patients receiving $5-10 \mathrm{mg} / \mathrm{d}$, to 1.85 for those receiving $20 \mathrm{mg} / \mathrm{d}$ or more, compared with controls. ${ }^{9}$
In our study, $26.7 \%$ of patients with infection required hospital admission, and $7.3 \%$ died within 7 days of diagnosis. Furthermore, by considering periods of drug use and changes in dosage over time (i.e., through time-variant exposure variables), we found marked dose-response effects for current and recent cumulative glucocorticoid use. This is consistent with results of a nested case-control study, conducted in Canada among 1947 cases and 16207 controls who had rheumatoid arthritis and were aged 60 years and older, ${ }^{7}$ in which the risk of infections requiring hospital admission varied with dosage and treatment duration. In agreement with our findings, a study in the United Kingdom identified older age but not sex as a risk factor for septicemia, lower respiratory tract infections and herpes zoster in patients treated with oral glucocorticoids, compared with nonusers. ${ }^{13}$

The large sample allowed the investigation of dose responses for different infection types and relevant subgroups (e.g., by disease duration). The contemporary study period and data linkage across primary and secondary care databases allowed for a large unselected cohort of patients with uncommon diseases and assessment of risk for both serious and nonserious infections. To minimize length and time-dependent bias, ${ }^{22,23}$ the start and end of follow-up were unrelated to dates of prescribed glucocorticoids, and medication variables identified periods with and without treatment (i.e., time variant). 
Table 3: Association between time-variant prescribed oral glucocorticoids and incident infections

\section{Adjusted hazard ratio* $(95 \% \mathrm{Cl})$; infection type}

\begin{tabular}{|c|c|c|c|c|c|}
\hline Variable & All-cause & Bacterial & Viral & Parasitic & Fungal \\
\hline Current use & $1.49(1.44-1.53)$ & $1.70(1.60-1.80)$ & $1.53(1.44-1.62)$ & $1.50(1.32-1.71)$ & $1.48(1.39-1.57)$ \\
\hline Current daily dose per $5 \mathrm{mg}$ daily & $1.13(1.12-1.14)$ & $1.16(1.14-1.19)$ & $1.13(1.11-1.15)$ & $1.14(1.09-1.18)$ & $1.11(1.09-1.14)$ \\
\hline \multicolumn{6}{|l|}{ Current daily dose category, mg } \\
\hline Nonuse (Ref.) & 1.00 & 1.00 & 1.00 & 1.00 & 1.00 \\
\hline$>0.0-4.9$ & $1.39(1.34-1.45)$ & $1.58(1.43-1.75)$ & $1.47(1.34-1.62)$ & $1.41(1.14-1.74)$ & $1.46(1.34-1.59)$ \\
\hline $5.0-14.9$ & $1.46(1.41-1.52)$ & $1.64(1.51-1.78)$ & $1.46(1.34-1.59)$ & $1.42(1.21-1.68)$ & $1.41(1.31-1.53)$ \\
\hline $15.0-24.9$ & $1.66(1.55-1.77)$ & $1.95(1.71-2.23)$ & $1.78(1.54-2.06)$ & $1.87(1.43-2.43)$ & $1.62(1.42-1.85)$ \\
\hline$\geq 25$ & $2.30(2.13-2.49)$ & $3.02(2.51-3.63)$ & $2.28(1.89-2.74)$ & $2.40(1.70-3.40)$ & $2.10(1.74-2.52)$ \\
\hline Cumulative dose in last year (per $1000 \mathrm{mg}$ ) & $1.50(1.49-1.52)$ & $1.53(1.51-1.55)$ & $1.51(1.50-1.53)$ & $1.52(1.49-1.55)$ & $1.59(1.57-1.61)$ \\
\hline \multicolumn{6}{|l|}{ Cumulative dose in last year category, $\mathrm{mg}$} \\
\hline Nonuse (Ref.) & 1.00 & 1.00 & 1.00 & 1.00 & 1.00 \\
\hline$>0.0-959.9$ & $1.88(1.84-1.92)$ & $2.17(2.09-2.25)$ & $2.30(2.22-2.37)$ & $2.43(2.25-2.60)$ & $2.31(2.23-2.39)$ \\
\hline $960-3054.9$ & $2.50(2.47-2.54)$ & $2.85(2.78-2.92)$ & $2.95(2.88-3.02)$ & $3.12(2.97-3.26)$ & $2.98(2.91-3.04)$ \\
\hline$\geq 3055$ & $3.23(3.19-3.28)$ & $3.69(3.60-3.79)$ & $3.72(3.63-3.81)$ & $3.90(3.70-4.10)$ & $3.66(3.56-3.75)$ \\
\hline Total cumulative dose (per 1000 mg) & $1.01(1.00-1.01)$ & $1.02(1.01-1.02)$ & $1.01(1.00-1.01)$ & $1.01(1.00-1.02)$ & $1.00(1.00-1.01)$ \\
\hline \multicolumn{6}{|l|}{ Total cumulative dose category, mg } \\
\hline Nonuse (Ref.) & 1.00 & 1.00 & 1.00 & 1.00 & 1.00 \\
\hline$>0.0-959.9$ & $1.08(1.03-1.14)$ & $1.10(0.98-1.23)$ & $1.08(0.97-1.21)$ & $1.08(0.86-1.36)$ & $1.07(0.96-1.18)$ \\
\hline $960-3054.9$ & $1.01(0.97-1.06)$ & $1.08(0.98-1.20)$ & $0.96(0.87-1.05)$ & $1.00(0.81-1.23)$ & $1.07(0.97-1.17)$ \\
\hline $3055-7299.9$ & $1.05(1.00-1.09)$ & $1.10(1.00-1.21)$ & $1.06(0.97-1.16)$ & $1.08(0.88-1.32)$ & $1.02(0.93-1.13)$ \\
\hline$\geq 7300$ & $1.11(1.06-1.17)$ & $1.36(1.23-1.51)$ & $1.13(1.02-1.25)$ & $1.17(0.94-1.47)$ & $1.07(0.96-1.19)$ \\
\hline
\end{tabular}

Note: $\mathrm{Cl}=$ confidence interval, Ref. = reference category.

*Hazard ratios from Cox proportional models adjusted for baseline age, sex, index of multiple deprivation, smoking status, ethnicity, body mass index, chronic inflammatory disease type, comorbidities (cardiovascular disease, diabetes, HIV, cancer, asthma, chronic obstructive pulmonary disease and renal disease), vaccination status (varicella zoster,

pneumococcus and influenza), number of hospital admissions in last year, prescribed non-oral glucocorticoids, proton pump inhibitors and $\mathrm{H}_{2}$ antagonists, and time-variant use of disease-modifying antirheumatic drugs and nonsteroidal antiinflammatory drugs; the practice identifier was included as a random intercept to account for clustering effect.

The observed high excess infection risk and infectionrelated mortality in patients with polymyalgia rheumatica or giant cell arteritis, even for daily doses of less than $5 \mathrm{mg}$, and the high dose-response estimates in users of non-oral glucocorticoids and vaccinated patients highlight the need for regular review of glucocorticoid requirements, even at low doses. Patients and clinicians should be educated about the risk of infection, need for symptom identification, prompt treatment, timely vaccination and documentation of history of chronic infection (e.g., herpes zoster).

\section{Limitations}

To define incident episodes of infection and its types, we used an algorithm combining diagnostic codes for infection and antibiotic and antiviral drug use. Reporting bias related to a higher suspicion of infections during periods of glucocorticoid use cannot be excluded. However, in our cohort, 93.7\% of infections were diagnosed on dates on which oral glucocorticoids were not prescribed, suggesting the primary reason for consultation was new infection-related symptoms. It remains likely that self-treated minor infections were missed.
No information on adherence or on hospital-prescribed medication was available, and missing glucocorticoid dose during tapering periods was imputed. This might underestimate medication use when patients are treated by specialists (e.g., around diagnosis or serious disease flares). Nevertheless, the median time since diagnosis was 5 years, a low proportion of patients had newly diagnosed polymyalgia rheumatica or giant cell arteritis, and the bulk of prescribing in the UK happens in primary care.

\section{Conclusion}

In this large, contemporary population-based study, we estimated absolute and relative risks of all-cause, bacterial, viral, parasitic and fungal infections and found strong glucocorticoid dose responses for all types among people diagnosed with polymyalgia rheumatica or giant cell arteritis. The estimates of dose response can be used to conduct benefit-harm evaluations and cost-effectiveness studies of new glucocorticoidsparing drugs required to guide policy and improve patient care outcomes in patients with polymyalgia rheumatica or giant cell arteritis. 
Table 4: Kaplan-Meier estimates of infections per level of time-variant current daily and cumulative oral glucocorticoid prednisolone-equivalent dose by time since diagnosis of polymyalgia rheumatica or giant cell arteritis

Cumulative probability, $\%(95 \% \mathrm{CI})^{*}$; time since diagnosis

Variable
All-cause infections
Incident infections, no. (\%)
At 1 year
Current daily dose, mg
Nonuse
$>0.0-4.9$
$5.0-14.9$
$15.0-24.9$
$\geq 25.0$

\section{Bacterial infections}

Incident infections, no. (\%)

\section{At 1 year}

Within 1 year

First year

$15187(54.2)$

$18.2(17.8-18.7)$

$1703(62.7)$

$18.0(16.6-19.5)$

$11.0(10.4-11.7)$

$17.3(16.4-18.3)$

$20.8(20.0-21.6)$

$31.2(29.1-33.2)$

$35.7(32.5-38.8)$

$13.0(11.0-15.0)$

$19.2(15.8-22.5)$

$22.2(19.2-25.1)$

27.5 (19.6-34.6)

$26.1(12.7-37.4)$

3421 (12.2)

$2.9(2.7-3.1)$

Current daily dose, $\mathrm{mg}$

$$
\begin{aligned}
& \text { Nonuse } \\
& >0.0-4.9 \\
& 5.0-14.9 \\
& 15.0-24.9 \\
& \geq 25.0
\end{aligned}
$$

\section{Viral infections}

Incident infections, no. (\%)

\section{At 1 year}

Current daily dose, $\mathrm{mg}$

$$
\begin{aligned}
& \text { Nonuse } \\
& >0.0-4.9 \\
& 5.0-14.9 \\
& 15.0-24.9 \\
& \geq 25.0
\end{aligned}
$$

\section{Parasitic infections}

Incident infections, no. (\%)

\section{At 1 year}

$1.4(1.2-1.7)$

$3.0(2.6-3.5)$

$3.3(2.9-3.6)$

$6.3(5.1-7.4)$

$7.5(5.7-9.4)$

$422(15.5)$
$3.1(2.5-3.8)$
$2.0(1.2-2.8)$
$3.6(1.9-5.2)$
$4.0(2.6-5.4)$
$6.3(2.0-10.5)$
$4.2(0.0-9.8)$

3731 (13.3)

$3.9(3.6-4.1)$

$2.2(1.9-2.5)$

$3.6(3.1-4.1)$

$4.6(4.2-5.1)$

$7.3(6.1-8.5)$

$8.4(6.4-10.3)$

361 (13.3)

$3.3(2.6-4.0)$

$2.1(1.2-2.9)$

$4.0(2.3-5.8)$

$4.5(3.0-6.0)$

$5.6(1.5-9.6)$

$2.2(0.0-6.3)$

$771(2.8)$

$1.0(0.8-1.1)$

Current daily dose, $\mathrm{mg}$

$$
\begin{aligned}
& \text { Nonuse } \\
& >0.0-4.9 \\
& 5.0-14.9 \\
& 15.0-24.9 \\
& \geq 25.0
\end{aligned}
$$

\section{Fungal infections}

Incident infections, no. (\%)

\section{At 1 year}

Current daily dose, mg

$$
\begin{aligned}
& \text { Nonuse } \\
& >0.0-4.9 \\
& 5.0-14.9 \\
& 15.0-24.9 \\
& \geq 25.0
\end{aligned}
$$

Note: $\mathrm{Cl}=$ confidence interval.

${ }^{*}$ Unless stated otherwise.

$0.7(0.4-1.0)$

$0.6(0.4-0.8)$

$0.3(0.0-0.5)$

$1.1(0.9-1.4)$

$1.2(0.4-1.9)$

$1.9(1.3-2.6)$

$3.0(0.1-5.9)$

$2.3(1.2-3.4)$

3890 (13.9)

351 (12.9)

$2.3(2.0-2.6)$

$1.7(1.0-2.5)$
$7.8(6.5-9.0)$

$9.4(7.3-11.4)$
$5.4(1.4-9.3)$

$2.0(0.0-5.9)$

Second year Third year

After third year

$1015(62.3)$
$18.8(16.8-20.7)$
$13.5(11.0-15.9)$
$20.2(15.2-25.0)$
$24.6(20.5-28.5)$
$28.2(16.8-38.0)$
$28.6(9.5-43.6)$

$807(61.6)$
$8.0(15.8-20.1)$

$3522(56.3)$

$18.7(17.7-19.7)$

14.1 (11.4-16.7)

$16.8(15.6-18.0)$

22.2 (16.6-27.5)

19.8 (17.0-22.4)

$20.2(15.4-24.7)$

21.6 (19.1-24.0)

$24.4(17.3-30.9)$

$26.5(9.9-40.0)$

$42.0(29.3-52.4)$

$254(15.6)$

$3.0(2.1-3.8)$

$227(17.3)$
$3.6(2.5-4.6)$

$910(14.5)$

$4.3(3.8-4.9)$

$1.8(0.9-2.7)$

$3.0(1.7-4.3)$

$3.6(3.0-4.2)$

$3.8(1.5-6.1)$

3.1 (1.4-4.8)

$5.7(2.5-8.7)$

$3.0(1.1-5.0)$

$9.7(2.6-16.4)$

$2.7(0.0-7.8)$

$5.7(4.1-7.2)$

$4.9(3.6-6.2)$

$6.2(2.4-9.9)$

$8.4(0.0-18.8)$

$10.0(0.0-22.2)$

15.0 (5.4-23.6)

$$
240 \text { (14.7) }
$$

$4.1(3.1-5.1)$

193 (14.7)

819 (13.1)

$3.8(2.7-4.9)$

$3.3(2.8-3.8)$

$3.2(2.0-4.4)$

3.2 (1.9-4.5)

$2.8(2.3-3.4)$

$5.7(2.9-8.5)$

$3.9(1.2-6.6)$

$2.4(1.3-3.4)$

$4.3(2.3-6.3)$

$4.1(1.8-6.4)$

$4.7(3.5-6.0)$

$2.7(0.0-7.8)$

$9.1(4.4-13.5)$

$4.2(0.0-11.8)$

$20.0(0.4-35.7)$

$3.5(0.0-8.3)$

40 (2.5)

$1.0(0.5-1.4)$

54 (4.1)

$182(2.9)$

$1.1(0.5-1.7)$

$0.8(0.6-1.0)$

$0.6(0.1-1.2)$

$1.0(0.3-1.8)$

$1.0(0.0-2.3)$

$1.1(0.0-2.3)$

$1.5(0.0-3.0)$

$1.0(0.0-1.9)$

$2.6(0.0-6.0)$

$0.0(0.0-0.0)$

$5.3(0.0-12.1)$

$0.0(0.0-0.0)$

$0.7(0.4-0.9)$

$0.9(0.2-1.5)$

$1.2(0.5-1.8)$

$0.6(0.0-1.7)$

$1.7(0.0-5.0)$

226 (13.9)

177 (13.5)

772 (12.3)

$4.0(3.0-5.0)$

$2.6(1.7-3.5)$

$3.2(2.8-3.7)$

$3.2(2.0-4.5)$

$2.2(1.1-3.3)$

$3.0(2.4-3.5)$

$4.2(1.7-6.6)$

$4.2(1.5-6.9)$

$3.1(1.9-4.3)$

$4.8(2.7-6.8)$

$2.1(0.4-3.7)$

$3.7(2.6-4.8)$

$7.1(0.9-12.9)$

$2.7(0.0-7.8)$

$5.0(1.5-8.3)$

$4.3(0.0-12.3)$

$5.3(0.0-14.8)$

$5.1(0.0-10.6)$ 


\section{References}

1. Dasgupta B, Borg FA, Hassan N, et al. BSR and BHPR guidelines for the management of giant cell arteritis. Rheumatology 2010;49:1594-7.

2. Dejaco C, Singh YP, Perel P, et al. 2015 Recommendations for the management of polymyalgia rheumatica: a European League Against Rheumatism/American College of Rheumatology collaborative initiative. Ann Rheum Dis 2015;74: 1799-807.

3. Boumpas DT, Chrousos GP, Wilder RL, et al. Glucocorticoid therapy for immune-mediated diseases: basic and clinical correlates. Ann Intern Med 1993; 119:1198-208.

4. Coutinho AE, Chapman KE. The anti-inflammatory and immunosuppressive effects of glucocorticoids, recent developments and mechanistic insights. Mol Cell Endocrinol 2011;335:2-13.

5. Purton JF, Monk JA, Liddicoat DR, et al. Expression of the glucocorticoid receptor from the $1 \mathrm{~A}$ promoter correlates with $\mathrm{T}$ lymphocyte sensitivity to glucocorticoidinduced cell death. J Immunol 2004;173:3816-24.

6. Youssef J, Novosad SA, Winthrop KL. Infection risk and safety of corticosteroid use. Rheum Dis Clin North Am 2016;42:157-76, ix-x.

7. Dixon WG, Abrahamowicz M, Beauchamp ME, et al. Immediate and delayed impact of oral glucocorticoid therapy on risk of serious infection in older patients with rheumatoid arthritis: a nested case-control analysis. Ann Rheum Dis 2012;71:1128-33.

8. Grijalva CG, Chen L, Delzell E, et al. Initiation of tumor necrosis factor-alpha antagonists and the risk of hospitalization for infection in patients with autoimmune diseases. JAMA 2011;306:2331-9.

9. Durand M, Thomas SL. Incidence of infections in patients with giant cell arteritis: a cohort study. Arthritis Care Res (Hoboken) 2012;64:581-8.

10. Wolfe F, Caplan L, Michaud K. Treatment for rheumatoid arthritis and the risk of hospitalization for pneumonia: associations with prednisone, disease-modifying antirheumatic drugs, and anti-tumor necrosis factor therapy. Arthritis Rheum 2006;54:628-34.

11. Widdifield J, Bernatsky S, Paterson JM, et al. Serious infections in a populationbased cohort of 86039 seniors with rheumatoid arthritis. Arthritis Care Res (Hoboken) 2013;65:353-61.
12. Schneeweiss S, Setoguchi S, Weinblatt ME, et al. Anti-tumor necrosis factor alpha therapy and the risk of serious bacterial infections in elderly patients with rheumatoid arthritis. Arthritis Rheum 2007;56:1754-64.

13. Fardet L, Petersen I, Nazareth I. Common infections in patients prescribed systemic glucocorticoids in primary care: a population-based cohort study. PLOS Med 2016;13:e1002024.

14. Herrett E, Gallagher AM, Bhaskaran K, et al. Data resource profile: Clinical Practice Research Datalink (CPRD). Int J Epidemiol 2015;44:827-36.

15. Mathur R, Bhaskaran K, Chaturvedi N, et al. Completeness and usability of ethnicity data in UK-based primary care and hospital databases. J Public Health (Oxf) 2014;36:684-92.

16. Hospital Episode Statistics (HES). UK: NHS Digital; updated 2019 Mar. 26. Available: https://digital.nhs.uk/data-and-information/data-tools-and-services/data-services/ hospital-episode-statistics (accessed 2018 Sept. 25).

17. Pujades-Rodriguez M, Duyx B, Thomas SL, et al. Associations between polymyalgia rheumatica and giant cell arteritis and 12 cardiovascular diseases. Heart 2016;102:383-9.

18. Smeeth L, Cook C, Hall AJ. Incidence of diagnosed polymyalgia rheumatica and temporal arteritis in the United Kingdom, 1990-2001. Ann Rheum Dis 2006;65:1093-8

19. Movahedi M, Costello R, Lunt M, et al. Oral glucocorticoid therapy and all-cause and cause-specific mortality in patients with rheumatoid arthritis: a retrospective cohort study. Eur J Epidemiol 2016;31:1045-55.

20. Noble M, McLennan D, Wiilkinson K, et al. The English indices of deprivation, 2007: technical report. London (UK): Ministry of Housing, Communities and Local Government; 2008.

21. Dixon WG, Suissa S, Hudson M. The association between systemic glucocorticoid therapy and the risk of infection in patients with rheumatoid arthritis: systematic review and meta-analyses. Arthritis Res Ther 2011;13:R139.

22. Lévesque LE, Hanley JA, Kezouh A, et al. Problem of immortal time bias in cohort studies: example using statins for preventing progression of diabetes. BMJ 2010;340:b5087.

23. Wolkewitz M, Allignol A, Harbarth S, et al. Time-dependent study entries and exposures in cohort studies can easily be sources of different and avoidable types of bias. J Clin Epidemiol 2012;65:1171-80.

\begin{abstract}
Competing interests: Christian Mallen and Ann Morgan received salary support from the National Institute for Health Research and the UK Medical Research Council. Ann Morgan has undertaken consultancy work in relation to giant cell arteritis for Roche, Chugai, GlaxoSmithKline, Sanofi and Regeneron Pharmaceuticals, with all funding paid directly into a research account. No other competing interests were declared.
\end{abstract}

This article has been peer reviewed.

Affiliations: School of Dentistry (Wu), University of Leeds; Leeds Institute for Data Analytics (Keeley, Morgan, Pujades-Rodriguez), University of Leeds, Leeds, UK; Arthritis Research UK Primary Care Centre (Mallen), University of Keele, Staffordshire, UK; Leeds Institute of Cardiovascular and Metabolic Medicine (Morgan), School of Medicine, University of Leeds; NIHR Leeds Biomedical Research Centre (Morgan), Leeds Teaching Hospitals NHS Trust; Leeds Institute of Health Sciences (Pujades-Rodriguez), School of Medicine, University of Leeds, Leeds, UK
Contributors: Jianhua Wu and Mar PujadesRodriguez had full access to all of the data in the study and take responsibility for the integrity of the data and the accuracy of the data analysis. Jianhua Wu and Mar PujadesRodriguez equally contributed to the work. Mar Pujades-Rodriguez and Ann Morgan conceived the study; Mar Pujades-Rodriguez designed the study, obtained ethics approval and acquired data; Mar Pujades-Rodriguez, Christian Mallen and Ann Morgan created and validated lists of diagnostic codes and algorithms to define infections; Mar PujadesRodriguez and Adam Keeley created disease cohorts and covariates; Jianhua Wu and Mar Pujades-Rodriguez managed, analyzed and interpreted data; Mar Pujades-Rodriguez drafted the manuscript; Jianhua Wu, Christian Mallen and Ann Morgan critically revised the manuscript for important intellectual content. All authors gave final approval of the version to be published and agreed to be accountable for all aspects of the work.

Funding: Jianhua Wu is supported by the National Institute for Health Research (NIHR) infrastructure at Leeds. Ann Morgan is supported by the Medical Research Council TARGET (Treatment According to Response in Giant Cell Arteritis) Partnership Grant (MR/ N011775/1) and the NIHR infrastructure at Leeds, including the NIHR Biomedical Research Centres (IS-BRC-1215-20015) and NIHR Medtech and In vitro diagnostics Cooperatives (MIC-2016-015). Christian Mallen is funded by an NIHR Research Professorship (NIHR-RP-2014-04-026), the NIHR Collaborations for Leadership in Applied Health Research and Care West Midlands and the NIHR School for Primary Care Research. The views expressed are those of the authors and not necessarily those of the NHS, the NIHR or the Department of Health and Social Care. The study funders had no role in the study design, data collection, analysis or interpretation, in the writing of the paper or in the decision to submit the paper for publication.

Accepted: May 24, 2019

Correspondence to: Mar Pujades-Rodriguez, M.D.M.PujadesRodriguez@leeds.ac.uk 